\title{
RESTRICTING THE ROST INVARIANT TO THE CENTER
}

\author{
S. GARIBALDI AND A. QUÉGUINER-MATHIEU
}

\begin{abstract}
For simple simply connected algebraic groups of classical type, Merkurjev, Parimala, and Tignol gave a formula for the restriction of the Rost invariant to the torsors induced from the center of the group. This paper completes their results by giving formulas for the exceptional groups. The method is somewhat different and also recovers their formula for classical groups.
\end{abstract}

\section{$\S 1$. INTRODUCTION}

In the 1990s, M. Rost proved that the group of degree 3 normalized invariants of an absolutely simple simply connected algebraic group $G$ over $k$, that is, the group of natural transformations of the Galois cohomology functors

$$
H^{1}(\star, G) \rightarrow H^{3}(\star, \mathbb{Q} / \mathbb{Z}(2)),
$$

is a cyclic group with a canonical generator. This canonical generator is known as the Rost invariant. Roughly speaking, it is the "first" nonzero invariant, in which there are no nonzero normalized invariants $H^{1}(\star, G) \rightarrow H^{d}(\star, \mathbb{Q} / \mathbb{Z}(d-1))$ for $d<3$ [KMRT, §31].

In general, there is no explicit formula for computing the Rost invariant. However, in [MPT], Merkurjev, Parimala, and Tignol gave a nice description, for $G$ of classical type, of the restriction of the Rost invariant to $H^{1}(\star, Z)$, where $Z$ is the center of $G$. Our purpose in this paper is to complete their results by providing a similar description for exceptional groups (see Theorem 2.3 for a precise statement for all types of groups).

Part of our proof is borrowed from [MPT]. Precisely, their Corollaries 1.2 to 1.6, which are stated for a general cycle module, show in our context that every group invariant

$$
H^{1}(\star, Z) \rightarrow H^{3}(\star, \mathbb{Q} / \mathbb{Z}(2))
$$

(i.e., every invariant such that the map $H^{1}(K, Z) \rightarrow H^{3}(K, \mathbb{Q} / \mathbb{Z}(2))$ is a group homomorphism for every extension $K / k)$ is given by some cup product with a class $t \in H^{2}(k, Z)$; see Proposition 2.1 and $\S 3$ below. (Note that this class $t$ does depend on a choice regarding the cup product; see Remark 2.5(iii).)

It remains to compute the class $t_{R, G} \in H^{2}(k, Z)$ associated with the composition

$$
H^{1}(\star, Z) \longrightarrow H^{1}(\star, G) \stackrel{r_{G}}{\longrightarrow} H^{3}(\star, \mathbb{Q} / \mathbb{Z}(2)),
$$

where the first map is induced by the inclusion of $Z$ in $G$ and $r_{G}$ is the Rost invariant of $G$. Note that, even though it is not obvious from the definition, this composition is actually a group invariant; see [Ga 01b, 7.1] or [MPT, Corollary 1.8]. The Rost invariant is canonically determined; nevertheless, practically speaking, one only knows the group it generates. Consequently, we will determine the subgroup $\left\langle t_{R, G}\right\rangle$ of $H^{2}(k, Z)$ generated by $t_{R, G}$.

2000 Mathematics Subject Classification. Primary 12G05.

Key words and phrases. Algebraic groups of classical type, exceptional groups, Rost invariant. 
For any semisimple group $G$, one has the Tits class $t_{G}$, an element of the group $H^{2}(k, Z)$. From [MPT] and from the present paper it follows that either $t_{R, G}$ is zero, or $t_{R, G}$ and $t_{G}$ generate the same subgroup of $H^{2}(k, Z)$, for a well-chosen cup product. Note that our argument for determining $\left\langle t_{R, G}\right\rangle$ is quite different from that in [MPT], where the authors used concrete interpretations of the classical groups. Here, we first reduce to groups whose Tits index satisfies a certain condition (see (4.1)), by using an injectivity result that follows from [MT, Theorem B]. Second, we prove the result for those particular groups, by reducing to groups of inner type $A$ for which the Rost invariant has a concrete description. This method applies to all absolutely simple groups except those of outer type $A$, and therefore can be used to recover the results of [MPT] on classical groups (except for their Theorem 1.10). We give the details for the types $B, C$, and $D_{\text {even }}$, as well as for the exceptional groups.

\section{§2. Statement of Results}

Throughout the paper, $G$ denotes an absolutely simple simply connected algebraic group over a field $k$. We assume (except in $\S 6$ ) that the characteristic of $k$ does not divide the exponent $n$ of the center of $G$. In other words, the characteristic is not 2 for $G$ of type $B, C, D, E_{7}$; the characteristic is not 3 for $G$ of type $E_{6}$; and the characteristic does not divide $\ell+1$ for $G$ of type $A_{\ell}$. This guarantees that the (scheme-theoretic) center $Z$ of $G$ is smooth. (See Remark 4.4 for comments on the characteristic hypothesis.)

The group $H^{d}(k, \mathbb{Q} / \mathbb{Z}(d-1))$ is as defined in [M 03, Appendix A]; otherwise the notation $H^{d}(k, A)$ stands for the Galois cohomology group $H^{d}\left(\Gamma_{k}, A\left(k_{\text {sep }}\right)\right)$, where $\Gamma_{k}$ denotes the Galois group of a separable closure $k_{\text {sep }}$ of $k$ over $k$ and $A$ is a smooth algebraic group. Our hypothesis on the characteristic of $k$ implies that the $n$-torsion of $H^{d}(k, \mathbb{Q} / \mathbb{Z}(d-1))$ is naturally identified with $H^{d}\left(k, \boldsymbol{\mu}_{n}^{\otimes(d-1)}\right)$ for $d=2,3$. If $G$ is not of type $A_{\ell}$ with $\ell \geq 3$ (e.g., in all cases studied in detail below), then $n$ is 2 or 3 ; hence $\boldsymbol{\mu}_{n}^{\otimes 2}$ is isomorphic to $\mathbb{Z} / n \mathbb{Z}$ (see [KMRT, p. 444]).

For any functor $\mathcal{F}$ from the category of field extensions of $k$ to the category of groups, we let $\operatorname{Inv}^{3}(\mathcal{F})$ be the collection of group invariants of $\mathcal{F}$ with values in $H^{3}(\star, \mathbb{Q} / \mathbb{Z}(2))$; this is an Abelian group. If $T$ is a quasitrivial torus, i.e., $T=R_{E / k}\left(\mathbb{G}_{m}\right)$ for some étale algebra $E / k$, then Theorem 1.1 of [MPT] implies that $H^{2}(k, T)$ is isomorphic to $\operatorname{Inv}^{3}(T)$. Precisely, the invariant $\alpha^{E}(X)$ associated with a cohomology class $X \in H^{2}(k, T)$ is defined by $\alpha^{E}(X)(y)=N_{E_{K} / K}\left(y \cdot X_{K}\right)$ for every field extension $K / k$ and $y \in T(K)=$ $E_{K}^{\times}$. The cup product appearing in this formula is given by the module structure on $H^{\star}(\mathbb{Q} / \mathbb{Z}(-1))$ over the Milnor $K$-ring (see [M 03, Appendix A] for the definition). Using this, we can prove the following.

Proposition 2.1. The groups $\operatorname{Inv}^{3}\left(H^{1}(\star, Z)\right)$ and $H^{2}(k, Z)$ are (noncanonically) isomorphic.

While proving this proposition in $\S 3$, we shall exhibit such isomorphisms; they will admit the following explicit description: for any cohomology class $t \in H^{2}(k, Z)$, the corresponding invariant is given by

$$
y \in H^{1}(K, Z) \mapsto y \cdot t_{K} \in H^{3}(K, \mathbb{Q} / \mathbb{Z}(2)) .
$$

Being a group invariant, its image is contained in the $n$-torsion, $H^{3}\left(K, \boldsymbol{\mu}_{n}^{\otimes 2}\right)$, of the group $H^{3}(K, \mathbb{Q} / \mathbb{Z}(2))$. Next, the cup product is induced by a bilinear form $Z\left(k_{\text {sep }}\right) \times Z\left(k_{\text {sep }}\right) \rightarrow$ $\boldsymbol{\mu}_{n}^{\otimes 2}$, which needs to be specified.

We denote by $\boldsymbol{\mu}_{n}$ the algebraic group of $n$th roots of unity and by $\boldsymbol{\mu}_{n[E]}$ the kernel of the norm map $N_{E / k}: R_{E / k}\left(\boldsymbol{\mu}_{n}\right) \rightarrow \boldsymbol{\mu}_{n}$, for any quadratic étale algebra $E / k$. As recalled in [MPT], the classification of the absolutely simple simply connected groups implies that 
if $G$ is classical, then $Z$ is one of the following groups: $\boldsymbol{\mu}_{n}, R_{E / k}\left(\boldsymbol{\mu}_{2}\right)$, or $\boldsymbol{\mu}_{n[E]}$, where $E$ is quadratic étale over $k$. If we wish to consider also exceptional groups, we need to add the centers of trialitarian $D_{4}$ groups, which are isomorphic to the kernel, now denoted by $R_{E / k}^{1}\left(\boldsymbol{\mu}_{2}\right)$, of the norm map $N_{E / k}: R_{E / k}\left(\boldsymbol{\mu}_{2}\right) \rightarrow \boldsymbol{\mu}_{2}$, where $E$ is cubic étale over $k$.

In most cases, namely, when $Z$ is $\boldsymbol{\mu}_{n}$ or $\boldsymbol{\mu}_{n[E]}$, there is a natural bilinear map

$$
Z\left(k_{\mathrm{sep}}\right) \times Z\left(k_{\mathrm{sep}}\right) \rightarrow \boldsymbol{\mu}_{n}^{\otimes 2} .
$$

The only groups for which the cup product must be defined carefully are groups of type $D_{\ell}$ with $\ell$ even. Their center is - over $k_{\text {sep }}$ - isomorphic to $\boldsymbol{\mu}_{2} \times \boldsymbol{\mu}_{2}$. Instead of fixing an identification between these two groups, we note that the fundamental weights of the root system give characters $\omega_{1}, \omega_{\ell-1}, \omega_{\ell}$ whose restrictions to $Z$ are the three nonzero homomorphisms $Z \rightarrow \boldsymbol{\mu}_{2}$. We consider the cup product induced by the following bilinear map with values in $\boldsymbol{\mu}_{2}^{\otimes 2}=\mathbb{Z} / 2 \mathbb{Z}$ :

$$
(x, y) \mapsto\left\{\begin{array}{lll}
\omega_{\ell-1}(x) \otimes \omega_{\ell}(y)+\omega_{\ell}(x) \otimes \omega_{\ell-1}(y) & \text { if } \ell \equiv 0 & (\bmod 4), \\
\omega_{\ell-1}(x) \otimes \omega_{\ell-1}(y)+\omega_{\ell}(x) \otimes \omega_{\ell}(y) & \text { if } \ell \equiv 2 & (\bmod 4) .
\end{array}\right.
$$

Since $\omega_{1}+\omega_{\ell-1}+\omega_{\ell}=0$ as characters, in the case where $\ell \equiv 0(\bmod 4)$ this cup product can be rewritten as

$$
(x, y) \mapsto \omega_{1}(x) \otimes \omega_{1}(y)+\omega_{\ell-1}(x) \otimes \omega_{\ell-1}(y)+\omega_{\ell}(x) \otimes \omega_{\ell}(y) .
$$

With this at hand, we can summarize the results of [MPT] and the present paper in the following theorem. ${ }^{1}$

Main Theorem 2.3. If $G$ is of type $A, C_{\ell}\left(\ell\right.$ odd), D, $E_{6}$, or $E_{7}$, then the composition (1.1) and the cup product with the Tits class of $G$ generate the same subgroup of $\operatorname{Inv}^{3}\left(H^{1}(\star, Z)\right)$. Otherwise the composition (1.1) is zero.

Example 2.4. For groups of inner type $A$ - that is, when $G$ is $S L(A)$ for some central simple algebra $A$ over $k$ - the theorem asserts that (1.1) and the cup product with the Brauer class [A] of $A$ generate the same subgroup of $\operatorname{Inv}^{3}\left(H^{1}(\star, Z)\right)$. This follows from the explicit description of the Rost invariant given in [M 03, p. 138]. This example is a starting point for proving the theorem.

The new cases in the theorem are the groups of types $E_{6}, E_{7}$, and trialitarian $D_{4}$, treated below in $\S \S 11,9$, and 12 , respectively. The exceptional groups of types $E_{8}, F_{4}$, and $G_{2}$ all have trivial center, so (1.1) is automatically zero for those groups.

Remark 2.5. (i) If the Tits class is zero, then a twisting argument shows that the composition (1.1) is zero, regardless of the type of $G$. Indeed, since the Tits class is zero, there is a simply connected quasisplit group $G^{q}$ such that we may identify $G$ with $G^{q}$ twisted by a 1-cocycle $\alpha \in Z^{1}\left(k, G^{q}\right)$. We write $Z^{q}$ for the center of $G^{q}$; the identification of $G$ with $G_{\alpha}^{q}$ identifies $Z$ with $Z^{q}$; we write $i$ and $i^{q}$ for the inclusions of $Z$ and $Z^{q}$ in $G$ and in $G^{q}$. The diagram

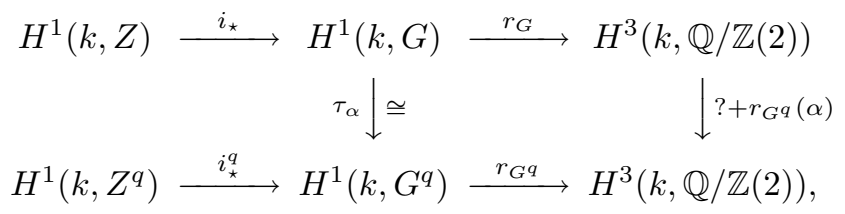

\footnotetext{
${ }^{1}$ There is a typo in Theorem 1.13 of [MPT], which addresses the $C_{\ell}$ case: the words "odd" and "even" should be interchanged. For keeping the $C_{\text {odd }}$ and $C_{\text {even }}$ cases straight, we find it helpful to recall that $B_{2}=C_{2} ;$ cf. $\S 10$ below.
} 
where $\tau_{\alpha}$ is the twisting isomorphism, commutes by [Gi, p. 76, Lemma 7] or [MPT, Corollary 1.8]. Fix $\zeta \in Z^{1}(k, Z)$. The image of $i_{\star} \zeta$ under $\tau_{\alpha}$ is the class in $H^{1}\left(k, G^{q}\right)$ of the 1-cocycle $\sigma \mapsto \zeta_{\sigma} \alpha_{\sigma}$ for $\sigma \in \Gamma_{k}$. But this is $\zeta \cdot \alpha \in H^{1}\left(k, G^{q}\right)$, where the - represents the usual action of $H^{1}\left(k, Z^{q}\right)$ on $H^{1}\left(k, G^{q}\right)$. Thus, the image of $i_{\star} \zeta$ in the lower right corner (when we go counterclockwise around the diagram) is $r_{G^{q}}(\zeta \cdot \alpha$ ), which equals $r_{G^{q}}\left(i_{\star}^{q} \zeta\right)+r_{G^{q}}(\alpha)$ by [Ga01b, 7.1]. But $G^{q}$ is quasisplit, so that it has a quasitrivial maximal torus $T^{q}$ that necessarily contains $Z^{q}$; hence, $i_{\star}^{q}$ factors through the zero group $H^{1}\left(k, T^{q}\right)$. We conclude that the image of $i_{\star} \zeta$ in the lower right corner is $r_{G^{q}}(\alpha)$.

On the other hand, going clockwise around the diagram, we find that the image of $i_{\star} \zeta$ in the lower right corner is $r_{G}\left(i_{\star} \zeta\right)+r_{G^{q}}(\alpha)$. The commutativity of the diagram proves the claim.

(ii) Note that the converse of (i) is not true in general. Indeed, if $G=\operatorname{Spin}(q)$ for some $(2 \ell+1)$-dimensional quadratic form $q$ over $k$, then the composition (1.1) is always zero. Meanwhile, the Tits class of $G$, which is the Brauer class of the even Clifford algebra of $q$, can be nonzero.

(iii) In the $D_{\ell}$ case with $\ell$ even, the bilinear form we use to compute the cup product does depend on whether $\ell / 2$ is even or odd. If we keep the same cup product in both cases, then the composition (1.1) coincides alternately with the cup product with $t_{G}$ and its conjugate.

(iv) The bilinear maps in (2.2) can be viewed as symmetric bilinear forms on a vector space of dimension 2 over the field with two elements. For $\ell \equiv 0(\bmod 4)$, it is the wedge product, or equivalently, a hyperbolic form. For $\ell \equiv 2(\bmod 4)$, it is a unique (up to isomorphism) metabolic form that is not hyperbolic.

\section{§3. INVARIANTS OF $H^{1}(\star, Z)$}

Our purpose in this section is to prove Proposition 2.1. In order to use the description of invariants of quasitrivial tori recalled above, we need to embed $Z$ in such a torus. More precisely, we are going to consider exact sequences

$$
1 \longrightarrow Z \stackrel{j}{\longrightarrow} T \stackrel{g}{\longrightarrow} T \longrightarrow 1,
$$

where $T$ is a quasitrivial torus, i.e., $T=R_{E / k}\left(\mathbb{G}_{m}\right)$ for some étale $k$-algebra $E$. We say that such an exact sequence is admissible if the map $g$ satisfies the following equation:

$$
N_{E_{K} / K}\left(g_{K}(y) \cdot X_{K}\right)=N_{E_{K} / K}\left(y \cdot H^{2}(g)(X)_{K}\right)
$$

for every $X \in H^{2}(k, T)$, every field extension $K / k$, and every $y \in T(K)=E_{K}^{\times}$.

We shall prove that any admissible exact sequence induces an isomorphism between $\operatorname{Inv}^{3}\left(H^{1}(\star, Z)\right)$ and $H^{2}(k, Z)$, as in Proposition 2.1. Indeed, we can apply [MPT, Lemma 3.1]: since the sequence

$$
T(K) \stackrel{g_{K}}{\longrightarrow} T(K) \stackrel{\varphi}{\longrightarrow} H^{1}(K, Z) \longrightarrow 1
$$

is exact for every extension $K / k$, the top row of the diagram

$$
\begin{aligned}
& 0 \longrightarrow \operatorname{Inv}^{3}\left(H^{1}(\star, Z)\right) \stackrel{\varphi^{\star}}{\longrightarrow} \operatorname{Inv}^{3}(T) \stackrel{g^{\star}}{\longrightarrow} \operatorname{Inv}^{3}(T) \\
& \alpha^{E} \uparrow \cong \alpha^{E} \uparrow \cong \\
& 1 \longrightarrow H^{2}(k, Z) \longrightarrow H^{2}(k, T) \stackrel{H^{2}(g)}{\longrightarrow} H^{2}(k, T)
\end{aligned}
$$

is also exact, where $\alpha^{E}$ is the isomorphism described in $\S 2$. Since $T$ is quasitrivial, the group $H^{1}(k, T)$ is zero and the bottom row of the diagram is also exact. An easy computation shows that under condition (3.1) the right-hand box is commutative. Since 
$\alpha^{E}$ is an isomorphism, this enables us to identify the kernels of both lines, producing an isomorphism $\beta$ as in the diagram.

To prove Proposition 2.1, it only remains to show that there exists an admissible exact sequence for the center $Z$ of a given $G$. This was done in [MPT] in Subsections 3.1 to 3.4 in the case where $Z$ is isomorphic to $\boldsymbol{\mu}_{n}, R_{E / k}\left(\boldsymbol{\mu}_{n}\right)$, or $\boldsymbol{\mu}_{n[E]}$ (see also the sequences $(3),(6)$, and (8)). The only remaining case is the center of a trialitarian $D_{4}$. For such a group, there exists a cubic field extension $E / k$ and an embedding $j: Z \rightarrow R_{E / k}\left(\mathbb{G}_{m}\right)$ that identifies $Z$ with the kernel $R_{E / k}^{1}\left(\mathbb{G}_{m}\right)$ of the norm map. Over $k_{\text {sep }}$, it is given by $j_{\text {sep }}(x)=\left(\omega_{1}(x), \omega_{\ell-1}(x), \omega_{\ell}(x)\right) \in\left(k_{\text {sep }}^{\times}\right)^{3}$.

We define $g: R_{E / k}\left(\mathbb{G}_{m}\right) \rightarrow R_{E / k}\left(\mathbb{G}_{m}\right)$ by $g_{K}(x)=N_{E_{K} / K}(x) x^{-2}$, for any field $K / k$ and any $x \in R_{E / k}\left(\mathbb{G}_{m}\right)(K)=E_{K}^{\times}$, and consider the sequence

$$
1 \longrightarrow Z \stackrel{j}{\longrightarrow} R_{E / k}\left(\mathbb{G}_{m}\right) \stackrel{g}{\longrightarrow} R_{E / k}\left(\mathbb{G}_{m}\right) \longrightarrow 1 .
$$

Lemma 3.4. Sequence (3.3) is exact and admissible.

Proof. An element $x$ in the kernel of $g_{K}$ satisfies $N_{E_{K} / K}(x)=x^{2}$. Since $E$ is cubic, taking the norm on both sides gives $1=N_{E_{K} / K}(x)=x^{2}$, proving that $x \in Z(K)$. The reader can easily check surjectivity; the admissibility condition 3.1 follows from the projection formula, as in [MPT, 3.3].

Now, Proposition 2.1 is proved.

Note that if you allow $E / k$ to be a cubic étale algebra rather than a field extension, then the sequence (3.3) is actually an admissible exact sequence for the center $Z$ of any group of type $D_{\ell}$ with $\ell \equiv 0 \bmod 4$. As we shall see in the next paragraph, the corresponding isomorphism between $\operatorname{Inv}^{3}\left(H^{1}(\star, Z)\right)$ and $H^{2}(k, Z)$ is not the same as that induced by the sequence (3) of [MPT] (see also Remark 2.5(iii)).

Hence, we need to specify which exact sequence we use: from now on, we shall always consider the isomorphism induced by the following admissible exact sequence, depending on $Z$ and $G$ :

- If $Z=\boldsymbol{\mu}_{n}$, we take

$$
1 \longrightarrow \boldsymbol{\mu}_{n} \longrightarrow \mathbb{G}_{m} \stackrel{n}{\longrightarrow} \mathbb{G}_{m} \longrightarrow 1 .
$$

- If $Z=\boldsymbol{\mu}_{n[E]}$ with $n$ odd (respectively, even), we take the sequence (6) (respectively, (8)) of [MPT].

- If $G$ is of type $D_{\ell}$ with $\ell \equiv 2 \bmod 4$, we take the sequence (3) of [MPT].

- If $G$ is of type $D_{\ell}$ with $\ell \equiv 0 \bmod 4$, we take the sequence (3.3).

In each of these cases, the isomorphism $\beta$ in (3.2) is given by

$$
\beta(t)(x)=x \cdot t_{K},
$$

where the cup product is induced by the bilinear map specified in $\S 2$. (This is the description of the isomorphism $\beta$ given immediately after the statement of Proposition 2.1.) This fact can be proved in each case by some explicit computation, going through the diagram. We do this for the groups of type $D_{\ell}$ with $\ell \equiv 0 \bmod 4$ and the sequence (3.3).

For any $t \in H^{2}(k, Z)$, the invariant $\beta(t)$ is characterized by the relation

$$
\beta(t)(\varphi(x))=\alpha^{E}\left(H^{2}(j)(t)\right)(x)=N_{E_{K} / K}\left(x \cdot H^{2}(j)\left(t_{K}\right)\right)
$$

for any $x \in T(K)$. We want to prove that this is equal to $\varphi(x) \cdot t_{K}$, where the cup product is induced by the bilinear map

$$
(x, y) \mapsto \omega_{1}(x) \otimes \omega_{1}(y)+\omega_{\ell-1}(x) \otimes \omega_{\ell-1}(y)+\omega_{\ell}(x) \otimes \omega_{\ell}(y) .
$$


Since $Z$ has exponent 2 , the map $j$ factors as

$$
Z \stackrel{j_{0}}{\longrightarrow} R_{E / k}\left(\boldsymbol{\mu}_{2}\right) \longrightarrow R_{E / k}\left(\mathbb{G}_{m}\right),
$$

and $j_{0_{\text {sep }}}$, as $j_{\text {sep }}$, coincides with the triple $\left(\omega_{1}, \omega_{\ell-1}, \omega_{\ell}\right)$. Hence, we have

$$
\varphi(x) \cdot t_{K}=N_{E_{K} / K}\left(H^{1}\left(j_{0}\right)(\varphi(x)) \cdot H^{2}\left(j_{0}\right)\left(t_{K}\right)\right) .
$$

To compare (3.6) and (3.7), we use the commutative diagram

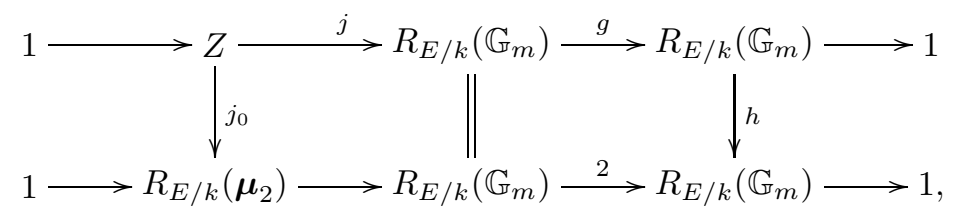

where the map $h$ is defined by the rule $h_{K}(x)=N_{E_{K} / K}(x) x^{-1}=x g_{K}(x)$ for any $x \in R_{E / k}\left(\mathbb{G}_{m}\right)(K)=E_{K}^{\times}$. It induces the commutative square

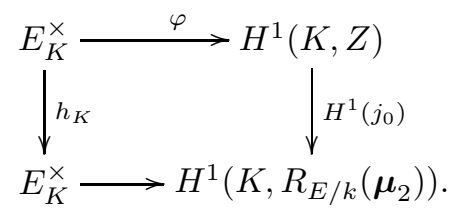

Consequently, $H^{1}\left(j_{0}\right)(\varphi(x))=\left(h_{K}(x)\right)_{2}=(x)_{2}+\left(g_{K}(x)\right)_{2}$, and

$$
\begin{aligned}
& N_{E_{K} / K}\left(H^{1}\left(j_{0}\right)(\varphi(x)) \cdot H^{2}\left(j_{0}\right)\left(t_{K}\right)\right) \\
& \quad=N_{E_{K} / K}\left((x)_{2} \cdot H^{2}\left(j_{0}\right)\left(t_{K}\right)\right)+N_{E_{K} / K}\left(\left(g_{K}(x)\right)_{2} \cdot H^{2}\left(j_{0}\right)\left(t_{K}\right)\right) \\
& \quad=N_{E_{K} / K}\left(x \cdot H^{2}(j)\left(t_{K}\right)\right)+N_{E_{K} / K}\left(g_{K}(x) \cdot H^{2}(j)\left(t_{K}\right)\right),
\end{aligned}
$$

where the cup product in the last line is given by the module structure on $H^{\star}(\mathbb{Q} / \mathbb{Z}(-1))$ over the Milnor $K$-ring (see [M03, Appendix A]). By the admissibility condition (3.1), the second term in the sum is

$$
N_{E_{K} / K}\left(x \cdot H^{2}(g) \circ H^{2}(j)\left(t_{K}\right)\right)=0,
$$

so that the expressions in (3.6) and (3.7) are equal. This finishes the proof of (3.5).

\section{§4. Reduction to groups having a particular Tits index}

For each group $G$, we let $t_{R, G}$ be the class in $H^{2}(k, Z)$ corresponding to the composition (1.1) under the isomorphism specified in the preceding section. The main theorem asserts that this class is zero or generates the same subgroup of $H^{2}(k, Z)$ as the Tits class $t_{G}$, depending on the type of the group $G$ we started with. A case by case proof will be given in $\S \S 7$ to 12 . First, we prove some general facts on which our strategy is based.

Given $G$, we fix a maximal $k$-split torus $S$, a maximal $k$-torus $T$ containing $S$, and a set of simple roots $\Delta$ of $G$ with respect to $T$. Recall the Tits index of $G$, as defined in [Ti66]. It is the data of the Dynkin diagram of $G$, together with the action of the Galois group $\operatorname{Gal}\left(k_{\mathrm{sep}} / k\right)$ on $\Delta$ via the $*$-action and the set $\Delta_{0}$ of $\alpha \in \Delta$ that vanish on $S$. The notation for $\Delta_{0}$ varies, but following [Ti 66], we circle a vertex if it belongs to $\Delta \backslash \Delta_{0}$, and circle together the vertices that are in the same Galois orbit.

Let $\Delta_{r}$ be the subset of $\Delta$ consisting of the simple roots such that the corresponding fundamental weight belongs to the root lattice. We consider the following condition on $G$ :

no $\alpha \in \Delta_{r}$ vanishes on $S$. 
In terms of Tits indices, this amounts to the following: every vertex in $\Delta_{r}$ is circled in the Tits index of $G$.

Dynkin diagrams for $E_{7}, E_{6}$, and $D_{\text {even }}$ with the vertices in $\Delta_{r}$ circled can be found in $\S \S 9,11$, and 12 below.

4.3. Now we give a method that reduces the proof of the Main Theorem 2.3 to the case where $G$ satisfies condition (4.2). Let $G$ be a simply connected absolutely simple group over $k$ and further suppose that $G$ is of inner type, i.e., that the absolute Galois group acts trivially on the Dynkin diagram of $G$. Since $G$ has inner type, the center $Z$ of $G$ is isomorphic to $\boldsymbol{\mu}_{n}$ or $\boldsymbol{\mu}_{2} \times \boldsymbol{\mu}_{2}$, and in $\S 3$ we fixed an injection $j: Z \rightarrow \mathbb{G}_{m}^{\times s}$ for $s \leq 3$.

Consulting the tables in [Bou], we observe that every element of $\Delta_{r}$ is fixed by every automorphism of the Dynkin diagram, hence is fixed by the $*$-action. It follows that the variety of parabolic subgroups of $G_{\text {sep }}$ of type $\Delta \backslash \Delta_{r}$ (in the notation of [BT, 4.2, 5.12] or [MT, p. 33]) is defined over $k$; see [BT, 5.24] or [BS, 8.4]. Since this variety has a point over its function field $F, G$ satisfies condition (4.2) over $F$. We have a commutative diagram with exact rows

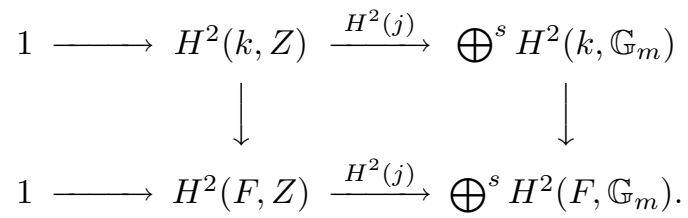

The kernel of the restriction map $H^{2}\left(k, \mathbb{G}_{m}\right) \rightarrow H^{2}\left(F, \mathbb{G}_{m}\right)$ was computed by Merkurjev and Tignol in [MT, Theorem B]. Let $\Lambda / \Lambda_{r}$ be the quotient of the weight lattice of $G$ by its root lattice. The kernel of the restriction map is generated by the Tits algebras associated with the classes in $\Lambda / \Lambda_{r}$ of the fundamental weights corresponding to the simple roots that belong to $\Delta_{r}$. But we have precisely chosen $\Delta_{r}$ so that these classes are zero. Hence, the restriction map $H^{2}\left(k, \mathbb{G}_{m}\right) \rightarrow H^{2}\left(F, \mathbb{G}_{m}\right)$ is injective. By the commutativity of the diagram, the map $H^{2}(k, Z) \rightarrow H^{2}(F, Z)$ is injective.

Let $t_{R, G}$ and the Tits class $t_{G} \in H^{2}(k, Z)$ be as defined in the Introduction. The isomorphism $\beta$ between $H^{2}(k, Z)$ and $\operatorname{Inv}^{3}\left(H^{1}(\star, Z)\right)$ (see $\left.\S 3\right)$ is functorial in $k$; i.e., the image of $t_{R, G}$ in $H^{2}(F, Z)$ is the element corresponding to the composition (1.1) over $F$. Therefore, if the composition (1.1) is zero over $F$, then $t_{R, G}$ is killed by $F$, and $t_{R, G}$ is zero in $H^{2}(k, Z)$; i.e., the composition (1.1) is zero over $k$. Alternatively, if the restrictions of $t_{R, G}$ and $t_{G}$ generate the same subgroup of $H^{2}(F, Z)$, then $t_{R, G}$ and $t_{G}$ generate the same subgroup of $H^{2}(k, Z)$.

In this way, we can reduce the proof of the main theorem for groups of inner type to the case of groups of inner type satisfying (4.2). This reduction can be generalized to treat also groups of outer type, but we shall not use this, and we omit it.

Remark 4.4. (i) For groups of type $A, \Delta_{r}$ is the empty set, so (4.2) is vacuously true, the function field $F$ is $k$ itself, and this reduction is useless.

(ii) Our global hypothesis excluding fields of certain characteristics arises from this argument, i.e., from Subsection 4.3. The hypothesis concerning the characteristic is needed for the identification of $H^{2}(k, Z)$ with $\operatorname{Inv}^{3}\left(H^{1}(\star, Z)\right)$ (see Proposition 2.1), because in the proof of that proposition we used [MPT, Theorem 1.1] to provide the isomorphism $\alpha^{E}$. In turn, the proof of the result from $[\mathrm{MPT}]$ requires that the $n$-torsion in $H^{3}(k, \mathbb{Q} / \mathbb{Z}(2))$ be a cycle module; in particular, one should be able to take residues relative to discrete valuations on $k$. This is only known for $n$ not divisible by char $k$.

With small changes, our proof for groups of inner type satisfying (4.2) works without any restriction on the characteristic of $k$. 


\section{$\S 5$. A SEMisimple SubGroup}

In this section, we assume that the group $G$ has Tits index satisfying condition (4.2). We produce a semisimple simply connected subgroup $G^{\prime}$ of $G$ that contains the center of $G$ and describe how to use $G^{\prime}$ in order to compute the Rost invariant of some elements of $G$.

5.1. Description of $G$ by generators and relations. We recall (see [St]) that $G$ is generated over $k_{\text {sep }}$ by the images of homomorphisms

$$
x_{\alpha}: \mathbb{G}_{a} \rightarrow G
$$

as $\alpha$ varies over the set of roots $\Phi$ of $G$ with respect to $T$. Write $\Lambda_{r}$ and $\Lambda$ for the root and weight lattices of $G$ with respect to $T$. Since $G$ is simply connected, $\Lambda$ is identified with the character group $T^{\star}=\operatorname{Hom}_{k_{\mathrm{sep}}}\left(T_{\mathrm{sep}}, \mathbb{G}_{m}\right)$ and $\Lambda_{r}$ with the character group $\bar{T}^{\star}$ of the image $\bar{T}$ of $T$ in $G / Z$.

We write $T_{\star}$ for the $\Gamma_{k}$-module of loops or cocharacters, i.e., $k_{\mathrm{sep}}$-homomorphisms $\mathbb{G}_{m} \rightarrow T_{\text {sep }}$. There is a natural pairing $T^{\star} \times T_{\star} \rightarrow \mathbb{Z}$ that enables us to identify $T_{\star}$ with the dual $\operatorname{Hom}(\Lambda, \mathbb{Z})$ of $T^{\star}$, i.e., with the lattice of coroots, denoted by $\Lambda_{r}^{\vee}$ (see [Bou, VI.1.1, Proposition 2]). We fix a set of simple roots $\Delta=\left\{\alpha_{1}, \alpha_{2}, \ldots, \alpha_{\ell}\right\}$ of $\Phi$; the coroots $\check{\alpha}_{1}, \check{\alpha}_{2}, \ldots, \check{\alpha}_{\ell}$ form a set of simple roots of the inverse root system $\Phi^{\vee}$ (see [Bou, VI.1.5, Remark 5]). For a given element $\check{\alpha}:=\sum c_{i} \check{\alpha}_{i}$ in $\Lambda_{r}^{\vee}$, the corresponding loop $\mathbb{G}_{m} \rightarrow T_{\text {sep }}$ is

$$
t \mapsto \prod h_{i}\left(t^{c_{i}}\right)
$$

where $h_{i}: \mathbb{G}_{m} \rightarrow T_{\text {sep }}$ is the loop corresponding to $\check{\alpha}_{i}$. The map $\prod h_{i}: \mathbb{G}_{m}^{\times \ell} \rightarrow T_{\text {sep }}$ is an isomorphism (see [St, p. 44, Corollary (a)]). We have the following identity:

$$
h_{\check{\alpha}}(t) x_{\beta}(u) h_{\check{\alpha}}(t)^{-1}=x_{\beta}\left(t^{(\beta, \check{\alpha})} u\right) \quad\left(\beta \in \Phi, \check{\alpha} \in \Phi^{\vee}\right) .
$$

5.4. Definition of $G^{\prime}$. Assume that $G$ satisfies (4.2). Let $\Lambda^{\vee}$ denote the weight lattice of the inverse root system; it has a basis dual to the basis $\Delta$ of $\Lambda_{r}$, and we write $\check{\omega}_{j}$ for the "coweight" such that $\left(\check{\omega}_{j}, \alpha_{i}\right)=\delta_{i j}$ (the Kronecker delta). We write $\bar{M}$ for the sublattice of $\Lambda^{\vee}$ generated by the $\check{\omega}_{j}$ for $\alpha_{j} \in \Delta_{r}$, and $M$ for the intersection $\bar{M} \cap \Lambda_{r}^{\vee}$. From [BT, 6.7, 6.9] it follows that $\Gamma_{k}$ acts trivially on $\bar{M}$, and hence also on $M$, because $\Delta_{r}$ is pointwise fixed by the Galois action. (Compare [M 96, 5.2].) Consequently, the loops in $M$ are $k$-homomorphisms $\mathbb{G}_{m} \rightarrow T$, and they generate a $k$-split torus $S^{\prime}$ of rank $\left|\Delta_{r}\right|$ in $G$.

We define $G^{\prime}$ to be the derived subgroup of the centralizer of $S^{\prime}$ in $G$.

Over $k_{\text {sep }}$, we can describe $G^{\prime}$ in specific terms. Identity (5.3) shows that $G^{\prime}$ is generated by the images of the $x_{\alpha}$ 's, where $\alpha$ varies over the roots in $\Phi$ whose support does not meet $\Delta_{r}$. In particular, $G^{\prime}$ is semisimple and even simply connected by [SS, 5.4b]. The Dynkin diagram of $G^{\prime}$ is obtained by deleting the vertices $\Delta_{r}$ from the Dynkin diagram of $G$. The intersection of $G^{\prime}$ with the maximal torus $T$ is, again over $k_{\text {sep }}$, the image of $\prod_{\alpha_{i} \notin \Delta_{r}} h_{i}$.

We are going to compute $t_{R, G}$ by reducing to this subgroup $G^{\prime}$. One reason why this is possible is the following fact.

Proposition 5.5. The center $Z$ of $G$ is contained in $G^{\prime}$.

Proof. It suffices to check this over an algebraic closure of $k$, so we may assume that $G^{\prime}$ and $G$ are split. Equation (5.3) implies that the center of $G$ is the subgroup of $T$ cut out 
by the roots; i.e., it is the intersection

$$
Z(G)=\bigcap_{\alpha \in \Phi} \operatorname{ker} \alpha
$$

Of course, we may replace the condition " $\alpha \in \Phi$ " with " $\alpha$ is in the root lattice". In particular, since the fundamental weight $\omega_{j}$ corresponding to $\alpha_{j} \in \Delta_{r}$ belongs to the root lattice, $Z(G)$ is contained in the kernel of $\omega_{j}$. Recall that $\omega_{j}$ is given by the formula

$$
\omega_{j}\left(\prod_{i} h_{i}\left(t_{i}\right)\right)=t_{j}
$$

Therefore, $Z(G)$ is a subgroup of $\prod_{\alpha_{i} \notin \Delta_{r}} h_{i}\left(\mathbb{G}_{m}\right)$. But this is the maximal torus of $G^{\prime}$.

5.6. Being simply connected, $G^{\prime}$ is of the form $G_{1}^{\prime} \times G_{2}^{\prime} \times \cdots \times G_{s}^{\prime}$, where each $G_{i}^{\prime}$ is simply connected and absolutely simple, and with Dynkin diagram a connected component of $\Delta \backslash \Delta_{r}$.

Lemma. An element $a \in Z^{1}\left(k, \prod_{i} G_{i}^{\prime}\right)$ can be written as $\prod_{i} a_{i}$ with $a_{i} \in Z^{1}\left(k, G_{i}^{\prime}\right)$, and the Rost invariant $r_{G}(a)$ equals $\sum_{i} m_{i} r_{G_{i}^{\prime}}\left(a_{i}\right)$, where $m_{i}$ is the Rost multiplier of the inclusion $G_{i}^{\prime} \subset G$.

Proof. We prove this by induction on the number $r$ of $a_{i}$ 's such that $a_{i}$ is not the zero cocycle in $H^{1}\left(k, G_{i}^{\prime}\right)$. The case where $r=1$ is trivial. We treat the general case. Let $i$ be such that $a_{i}$ is not zero. Consider the group $G_{a_{i}}$ obtained by twisting $G$ by $a_{i}$; since $G_{i}^{\prime}$ commutes with $G_{j}^{\prime}$ for $j \neq i$, the twisted group contains $\prod_{j \neq i} G_{j}^{\prime}$ in an obvious way. We find a diagram

$$
\begin{aligned}
& H^{1}\left(k, \prod_{j \neq i} G_{j}\right) \longrightarrow H^{1}\left(k, G_{a_{i}}\right) \stackrel{r_{G_{a_{i}}}}{\longrightarrow} H^{3}(k, \mathbb{Q} / \mathbb{Z}(2)) \\
& \tau_{a_{i}} \downarrow \\
& H^{1}(k, G) \stackrel{{ }^{\prime}}{\longrightarrow} \stackrel{r_{G}}{\longrightarrow} H^{3}(k, \mathbb{Q} / \mathbb{Z}(2)),
\end{aligned}
$$

where $\tau_{a_{i}}$ denotes the twisting map. This diagram commutes, as in Remark 2.5. Starting with $\prod_{j \neq i} a_{j}$ in the upper left, we find $\sum_{j \neq i} m_{j} r_{G_{j}^{\prime}}\left(a_{j}\right)$ in the upper right by the induction hypothesis and $a$ in $H^{1}(k, G)$ in the lower left. The commutativity of the diagram gives the desired equation.

5.7. We continue the notation of Subsection 5.6. We are mainly interested in the case where $G$ is "simply laced", i.e., where all roots have the same length. In that case, the inclusions $G_{i}^{\prime} \subset G$ all have Rost multiplier one (see, e.g., [Ga 01b, 2.2] or [M 03, 7.9.2]), and the formula in Lemma 5.6 simply says:

$$
r_{G}(a)=\sum_{i} r_{G_{i}^{\prime}}\left(a_{i}\right)
$$

\section{$\S 6$. The CEnTER AND THE TORUS}

In this section, the field $k$ will be arbitrary, with no restriction on the characteristic. We compute how the scheme-theoretic center $Z$ of a semisimple simply connected group $G$ sits inside a fixed maximal torus, in terms of the root system. We do this by using an exact sequence from [M 96], which we first recall.

6.1. Isogenies of tori. Let $T \rightarrow \bar{T}$ be a $k$-isogeny of tori with kernel $Z$; i.e., $Z$ is finite and the sequence

$$
1 \longrightarrow Z \longrightarrow T \stackrel{\pi}{\longrightarrow} \bar{T} \longrightarrow 1
$$


is exact. As before, $T_{\star}$ (respectively, $\bar{T}_{\star}$ ) denotes the $\Gamma_{k}$-module of loops or cocharacters, i.e., $k_{\text {sep }}$ homomorphisms $\mathbb{G}_{m} \rightarrow T_{\text {sep }}$ (respectively, $\mathbb{G}_{m} \rightarrow \bar{T}_{\text {sep }}$ ).

Proposition 6.2. There is a map $z$ such that

$$
1 \longrightarrow T_{\star} \stackrel{\pi_{\star}}{\longrightarrow} \bar{T}_{\star} \stackrel{z}{\longrightarrow} \lim _{\longrightarrow} \operatorname{Hom}_{k_{\mathrm{sep}}}\left(\boldsymbol{\mu}_{n}, Z_{\mathrm{sep}}\right) \longrightarrow 1
$$

is an exact sequence of $\Gamma_{k}$-modules.

This is sketched in $\S 1$ of Merkurjev's paper (loc. cit.). We give an explicit proof, including a precise description of $z$ that will be used later.

We start with the following lemma.

Lemma 6.3. The image of every $k$-homomorphism from $\boldsymbol{\mu}_{n}$ to a split torus $S$ is contained in a rank 1 subtorus of $S$.

Proof. We may assume that $\boldsymbol{\mu}_{n}$ injects into $S$. After dualization, such an injection corresponds to a surjection $\pi: \mathbb{Z}^{r} \rightarrow \mathbb{Z} / n \mathbb{Z}$, where $r$ is the rank of $S$. By the fundamental theorem for finitely generated Abelian groups, there is a basis $b_{1}, \ldots, b_{r}$ of $\mathbb{Z}^{r}$ such that the kernel of $\pi$ has the basis $b_{1}, b_{2}, \ldots, b_{r-1}, n b_{r}$. The projection to the $b_{r}$-coordinate yields a map $\pi_{r}: \mathbb{Z}^{r} \rightarrow \mathbb{Z}$, and $\pi$ factors through $\pi_{r}$. Another dualization gives the lemma.

Proof of Proposition 6.2. Since $\pi$ has finite kernel, the induced map $\pi_{\star}$ is injective. We use it to identify $T_{\star}$ with a submodule of $\bar{T}_{\star}$. Since the tori have the same rank, the quotient $\bar{T}_{\star} / T_{\star}$ is finite.

Now we define $z$. For $\omega \in \bar{T}_{\star}$, let $n$ be the smallest positive integer such that $n \omega$ belongs to $T_{\star}$. We define $z_{\omega}: \boldsymbol{\mu}_{n} \rightarrow T_{\text {sep }}$ to be the restriction of the loop $n \omega: \mathbb{G}_{m} \rightarrow T_{\text {sep }}$ to $\boldsymbol{\mu}_{n}$. Since every character $\chi$ of $\bar{T}$ maps $\bar{T}_{\star}$ to $\mathbb{Z}$, we have $\chi(n \omega) \in n \mathbb{Z}$, and the composition

$$
\boldsymbol{\mu}_{n} \stackrel{z_{\omega}}{\longrightarrow} T \longrightarrow \bar{T} \stackrel{\chi}{\longrightarrow} \mathbb{G}_{m}
$$

is 1 . Since the subgroups of $T$ are cut out by characters, the image of $z_{\omega}$ belongs to $Z$. It is an exercise to verify that the sequence displayed in the lemma is exact at $\bar{T}_{\star}$. Note that the map $z$ is $\Gamma_{k}$-equivariant.

It remains to prove that $z$ is surjective. Suppose $\phi: \boldsymbol{\mu}_{n} \rightarrow Z_{\text {sep }}$ is a homomorphism defined over $k_{\text {sep }}$. By Lemma 6.3, there is a rank 1 subtorus $S_{\text {sep }}$ of $T_{\text {sep }}$ such that the image of $\phi$ is contained in $S_{\text {sep }}$; let $\rho$ be a loop generating $S_{\star}$. By assumption, $\chi(\pi \rho)$ is in $n \mathbb{Z}$ for every character $\chi \in \bar{T}^{\star}$, so there is some $\omega \in \bar{T}_{\star}$ such that $\pi \rho=n \omega$. Then $\phi$ and $z_{\omega}$ agree in $\lim _{\longrightarrow} \operatorname{Hom}\left(\boldsymbol{\mu}_{n}, Z_{\mathrm{sep}}\right)$.

In our situation (with the notation of Subsections 5.1 and 5.4), the center $Z$ of $G$ is contained in the maximal torus $T$ and the sequence

$$
1 \longrightarrow Z \longrightarrow T \longrightarrow \bar{T} \longrightarrow 1
$$

is exact. For the same reason that $T_{\star}$ is identified with the coroot lattice $\Lambda_{r}^{\vee}$, the lattice $\bar{T}_{\star}$ is identified with $\Lambda^{\vee}$. Hence, the exact sequence given by Proposition 6.2 can be rewritten as

$$
1 \longrightarrow \Lambda_{r}^{\vee} \longrightarrow \Lambda^{\vee} \longrightarrow \lim _{n} \operatorname{Hom}_{k_{\text {sep }}}\left(\boldsymbol{\mu}_{n}, Z_{\text {sep }}\right) \longrightarrow 1 .
$$

We close this section with an application of this exact sequence. We shall only use it for groups of types $B$ and $C$, but we include it because of independent interest. Let $\Delta_{c}$ stand for the set of $\alpha_{j} \in \Delta$ such that $\check{\omega}_{j}$ (as defined in Subsection 5.4) is a minuscule weight for the inverse root system $\Phi^{\vee}$. Recall (see [Bou, §VI.1, Exercise 24]) that the minuscule weights are the minimal nonzero dominant weights. 
Corollary 6.5. If $G$ is of inner type and $\Delta_{c}$ does not meet $\Delta_{0}$, then the center $Z$ of $G$ is contained in a $k$-split subtorus $S$ of $T$.

The condition on $\Delta_{c}$ is the same as saying that every element of $\Delta_{c}$ is circled in the index of $G$.

Remark 6.6. In the situation of the corollary, $H^{1}(k, S)$ is zero, so that the natural map $H_{\text {fppf }}^{1}(k, Z) \rightarrow H^{1}(k, G)$ is zero. (If $Z$ is smooth, as in the rest of this paper, then the group $H_{\mathrm{fppf}}^{1}(k, Z)$ agrees with the Galois cohomology group $H^{1}(k, Z)$.) This has two useful consequences:

(i) the composition (1.1) is zero;

(ii) the connecting homomorphism $(G / Z)(k) \rightarrow H_{\mathrm{fppf}}^{1}(k, Z)$ is surjective.

Proof of Corollary 6.5. Since the minuscule weights generate $\Lambda^{\vee} / \Lambda_{r}^{\vee}$ (see [Bou, §VI.2, Exercise 5]), $Z$ is generated by the images of $z_{\breve{\omega}_{j}}$ for $\alpha_{j} \in \Delta_{c}$. Using the same argument as in the proof of Proposition 6.2, we see that $Z$ is contained in the torus $Q$ (defined over $k_{\text {sep }}$ ) corresponding to the $n_{j} \check{\omega}_{j}$ for $\alpha_{j} \in \Delta_{c}$, where $n_{j}$ is the smallest natural number such that $n_{j} \check{\omega}_{j}$ is in $\Lambda_{r}^{\vee}$.

Since $G$ has inner type, the maximal $k$-split torus $S$ in $T$ is the intersection of the kernels of $\alpha \in \Delta_{0}$ by [BT, 6.7, 6.9]. For such an $\alpha$ and for $\alpha_{j} \in \Delta_{c}$, we have $\alpha \neq \alpha_{j}$ by assumption; therefore, the inner product $\left(\alpha, \breve{\omega}_{j}\right)$ is zero. We conclude that the torus $Q$ is contained in $S$.

\section{$\S 7$. TYPE $B$}

Before proving the main theorem for exceptional groups, we show that our method gives a new and very short proof for groups of type $B_{\ell}(\ell \geq 2)$, i.e., that the composition (1.1) is zero. By Subsection 4.3, it suffices to prove this for groups with Tits index satisfying (4.2). Consulting the tables in [Bou], we see that

$$
\Delta_{r}=\left\{\alpha_{1}, \alpha_{2}, \ldots, \alpha_{\ell-1}\right\} .
$$

The inverse root system is $C_{\ell}$, and its only minuscule weight is $\check{\omega}_{1}$, i.e.,

$$
\Delta_{c}=\left\{\alpha_{1}\right\} .
$$

Since, by (4.2), the vertex $\alpha_{1}$ is circled in the index of $G$, Remark 6.6(i) shows that the composition (1.1) is zero.

Note that the groups of type $B_{\ell}$ satisfying the hypotheses of Corollary 6.5 are those of the form $\operatorname{Spin}(q)$, where $q$ is a $(2 \ell+1)$-dimensional isotropic quadratic form. For these groups, Remark 6.6(ii) says: the spinor norm map $S O(q)(k) \rightarrow k^{\times} / k^{\times 2}$ is surjective.

\section{§8. Concrete Description of the CEnTER}

We now give a concrete description of the center $Z$ inside a simply connected group $G$ in terms of the generators and relations for $G$ as in Subsection 5.1; consequently, we work over the separably closed field $k_{\text {sep }}$. (But see Subsection 8.10 below.) Note that, since the sequence (6.4) is exact, it suffices to compute $z_{\check{\omega}}$ for $\check{\omega}$ belonging to a set of representatives in $\Lambda^{\vee}$ of generators of the quotient group $\Lambda^{\vee} / \Lambda_{r}^{\vee}$.

Example 8.1 $\left(E_{7}\right)$. The center of a simply connected group $G$ of type $E_{7}$ is isomorphic to $\boldsymbol{\mu}_{2}$. Since all roots have the same length, we can normalize the Weyl-invariant inner product so that all roots have length 2; this identifies $\Phi$ with the inverse system $\Phi^{\vee}$. The fundamental weight

$$
\omega_{7}=\alpha_{1}+\frac{3}{2} \alpha_{2}+2 \alpha_{3}+3 \alpha_{4}+\frac{5}{2} \alpha_{5}+2 \alpha_{6}+\frac{3}{2} \alpha_{7}
$$


is not in the root lattice and, thus, maps to the nonidentity element in $\Lambda / \Lambda_{r}$. (We systematically number roots as in Bourbaki; for a diagram see §9.) The corresponding $\operatorname{map} z_{\omega_{7}}: \boldsymbol{\mu}_{2} \rightarrow Z_{\text {sep }}$ is given by

$$
z_{\omega_{7}}(-1)=h_{2 \omega_{7}}(-1)=h_{2}(-1) h_{5}(-1) h_{7}(-1) .
$$

Here we can see Proposition 5.5 explicitly: $\Delta \backslash \Delta_{r}$ is $\left\{\alpha_{2}, \alpha_{5}, \alpha_{7}\right\}$, and the image of $z_{\omega_{7}}$ is contained in $G^{\prime}$.

Example 8.2 $\left(E_{6}\right)$. Now, let $G$ be of type $E_{6}$. The center of $G$ is isomorphic to $\boldsymbol{\mu}_{3}$. Again we can identify $\Phi$ and $\Phi^{\vee}$. The fundamental weight

$$
\omega_{1}=\frac{4}{3} \alpha_{1}+\alpha_{2}+\frac{5}{3} \alpha_{3}+2 \alpha_{4}+\frac{4}{3} \alpha_{5}+\frac{2}{3} \alpha_{6}
$$

is not in the root lattice; so its class generates $\Lambda / \Lambda_{r}$. The corresponding isomorphism $z_{\omega_{1}}: \boldsymbol{\mu}_{3} \rightarrow Z_{\text {sep }}$ is defined by

$$
z_{\omega_{1}}(\zeta)=h_{3 \omega_{1}}(\zeta)=h_{1}(\zeta) h_{3}\left(\zeta^{2}\right) h_{5}(\zeta) h_{6}\left(\zeta^{2}\right)
$$

Example $8.4\left(C_{\ell}\right)$. Let $G$ be of type $C_{\ell}$; the center $Z$ is isomorphic to $\boldsymbol{\mu}_{2}$. In this case, we must be somewhat more careful because the root system has roots of different lengths. The inverse system $\Phi^{\vee}$ is $B_{\ell}$. We write $\check{\omega}_{\ell}$ for the fundamental weight corresponding to the root $\check{\alpha}_{\ell}$ in the inverse system, i.e.,

$$
\check{\omega}_{\ell}=\frac{1}{2}\left(\check{\alpha}_{1}+2 \check{\alpha}_{2}+\cdots+\ell \check{\alpha}_{\ell}\right)
$$

The corresponding isomorphism $\boldsymbol{\mu}_{2} \rightarrow Z_{\text {sep }}$ is given by

$$
z_{\check{\omega}_{\ell}}(-1)= \begin{cases}h_{2 \check{\omega}_{\ell}}(-1)=h_{1}(-1) h_{3}(-1) \cdots h_{\ell-1}(-1) & \text { if } \ell \text { is even } \\ h_{2 \check{\omega}_{\ell}}(-1)=h_{1}(-1) h_{3}(-1) \cdots h_{\ell}(-1) & \text { if } \ell \text { is odd. }\end{cases}
$$

Example 8.6 $\left(D_{\text {even }}\right)$. For $G$ of inner type $D_{\ell}$ with $\ell$ even, the center $Z$ is isomorphic to $\boldsymbol{\mu}_{2} \times \boldsymbol{\mu}_{2}$. As for $E_{7}$, all roots have the same length and we identify the root system with the inverse system. The fundamental weights

$$
\begin{aligned}
\omega_{\ell-1} & =\frac{1}{2} \alpha_{1}+\alpha_{2}+\frac{3}{2} \alpha_{3}+\cdots+\frac{\ell-2}{2} \alpha_{\ell-2}+\frac{\ell}{4} \alpha_{\ell-1}+\frac{\ell-2}{4} \alpha_{\ell}, \\
\omega_{\ell} & =\frac{1}{2} \alpha_{1}+\alpha_{2}+\frac{3}{2} \alpha_{3}+\cdots+\frac{\ell-2}{2} \alpha_{\ell-2}+\frac{\ell-2}{4} \alpha_{\ell-1}+\frac{\ell}{4} \alpha_{\ell}
\end{aligned}
$$

have two different residues in $\Lambda / \Lambda_{r}$. Since this is the Klein four-group, the two residues generate it. It follows that the images of the corresponding maps $z_{\omega_{\ell-1}}, z_{\omega_{\ell}}: \boldsymbol{\mu}_{2} \rightarrow Z_{\text {sep }}$

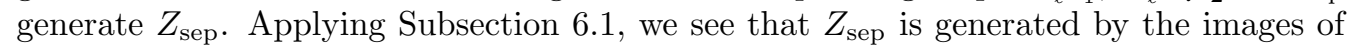
the homomorphisms $z_{0}, z_{1}$ defined by

$$
\begin{aligned}
& z_{0}(-1):=h_{1}(-1) h_{3}(-1) \cdots h_{\ell-3}(-1) h_{\ell}(-1), \\
& z_{1}(-1):=h_{1}(-1) h_{3}(-1) \cdots h_{\ell-3}(-1) h_{\ell-1}(-1) .
\end{aligned}
$$

Note that we have abandoned tying the weights with their corresponding homomorphism, because this depends on the parity of $\ell / 2$.

Example 8.8 $\left(A_{2}\right)$. Suppose now that $G$ has type $A_{2}$, i.e., $G$ is isomorphic to $S L_{3}$ and its center is isomorphic to $\boldsymbol{\mu}_{3}$. Since the fundamental weight $\omega_{1}=\frac{2}{3} \alpha_{1}+\frac{1}{3} \alpha_{2}$ does not belong to the root lattice, we get an isomorphism $z_{\omega_{1}}: \boldsymbol{\mu}_{3} \rightarrow Z_{\text {sep }}$ defined by

$$
z_{\omega_{1}}(\zeta)=h_{1}\left(\zeta^{2}\right) h_{2}(\zeta)
$$


8.10. Let $G$ be a simply connected semisimple group of inner type over $k$. Each element $\check{\omega}$ of $\Lambda^{\vee}$ determines an isomorphism

$$
z_{\breve{\omega}}: \boldsymbol{\mu}_{n} \rightarrow \boldsymbol{\mu}_{n} \subset G
$$

over $k_{\text {sep }}$. But the only morphisms $\boldsymbol{\mu}_{n} \rightarrow \boldsymbol{\mu}_{n}$ are raising to a power, so that $z_{\check{\omega}}$ is $\Gamma_{k}$-equivariant, and hence is defined over $k$ by [Borel, AG.14.3].

\section{§9. TYPE $E_{7}$}

In this section we prove the Main Theorem 2.3 for a group $G$ of type $E_{7}$. In this case, the center $Z$ is identified with $\boldsymbol{\mu}_{2}$. We prove that $t_{R, G}$ coincides with the Tits class $t_{G}$; i.e., the composition (1.1) is the cup product with $t_{G}$, where the cup product is induced by the obvious bilinear map $Z\left(k_{\mathrm{sep}}\right) \times Z\left(k_{\mathrm{sep}}\right)=\boldsymbol{\mu}_{2}\left(k_{\mathrm{sep}}\right) \times \boldsymbol{\mu}_{2}\left(k_{\mathrm{sep}}\right) \rightarrow \boldsymbol{\mu}_{2}\left(k_{\mathrm{sep}}\right)^{\otimes 2}$. (Since the group $H^{2}(k, Z)$ is 2-torsion, $t_{R, G}$ and $t_{G}$ generate the same subgroup of $H^{2}(k, Z)$ if and only if they are equal.)

By Subsection 4.3, it suffices to prove this for groups of type $E_{7}$ with Tits index satisfying (4.2). Let $G$ be such a group; i.e., assume that the index of $G$ is

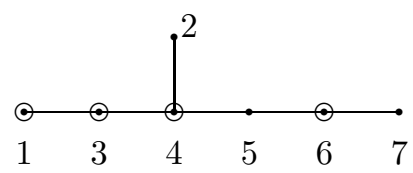

or possibly has more circled vertices. (If the index has more circles, then $G$ is split; see [Ti66, p. 57].)

We write $G_{i}^{\prime}$ with $i=2,5,7$ for the components of $G^{\prime}$, where $G_{i}^{\prime}$ corresponds to the vertex $\alpha_{i}$ in the Dynkin diagram. Each is isomorphic to $S L_{1}\left(Q_{i}\right)$ for some quaternion algebra $Q_{i}$. Since the weights $\omega_{2}, \omega_{5}, \omega_{7}$ all have the same image in $\Lambda / \Lambda_{r}$, the $Q_{i}$ are all isomorphic by [Ti71, p. 211] and we simply write $Q$. By [Ti71, loc. cit.], the class of $Q$ in $H^{2}\left(k, \mu_{2}\right)$ is also the Tits class of $G$. The center $Z$ is contained in the product $Z_{2} \times Z_{5} \times Z_{7}$ of the centers of $G_{2}^{\prime}, G_{5}^{\prime}, G_{7}^{\prime}$ by Proposition 5.5. By the description of the center given in Example 8.1, the map

$$
\boldsymbol{\mu}_{2}=Z \rightarrow Z_{2} \times Z_{5} \times Z_{7}=\boldsymbol{\mu}_{2} \times \boldsymbol{\mu}_{2} \times \boldsymbol{\mu}_{2}
$$

is $-1 \mapsto(-1,-1,-1)$. (Even though Example 8.1 treats $G$ over the separable closure, it still applies to our nonsplit $G$ over $k$ by Subsection 8.10.) Applying equation (5.8) and the type $A_{1}$ case of the main theorem (see Remark 2.4), we see that the class $t_{R, G}$ is $3[Q]=[Q]$, which is the Tits class of $G$.

This concludes the proof of the main theorem for groups of type $E_{7}$.

\section{$\S 10$. TYPE $C$}

The argument of the preceding section can be adapted to recover the theorem for groups of type $C_{\ell}$. (Alternatively, in the case where $\ell$ is even, the same argument as for type $B$ in $\S 7$ shows that the composition (1.1) is zero.) Since the result is not new for type $C$, we only briefly sketch the proof. We want to show that $t_{R, G}$ is 0 if $\ell$ is even and $t_{G}$ if $\ell$ is odd. Recall that a group of type $C_{\ell}$ has Dynkin diagram

$$
\underset{123}{\longrightarrow} \cdots \frac{\ell-1}{\longrightarrow}
$$


As before, it suffices to prove this for groups whose Tits index satisfies condition (4.2), i.e., such that $\alpha_{i}$ is circled for $i$ even.

As before, we write $G_{i}^{\prime}$ for the component of $G^{\prime}$ corresponding to the vertex $\alpha_{i}$, for $1 \leq i \leq n, i$ odd. We may identify all those with $S L(Q)$ for some quaternion algebra $Q$. The description of the center given in Example 8.4 shows that $Z$ maps to the product of the centers of the $G_{i}^{\prime}$ by $(-1) \mapsto(-1, \ldots,-1)$.

It remains to apply Lemma 5.6, which asserts that $r_{G}(a)=\sum_{i} m_{i} r_{G_{i}^{\prime}}\left(a_{i}\right)$. Note that we do have to care about Rost multipliers here, since the groups of type $C$ are not simply laced. If $1 \leq i \leq n-1, i$ odd, then the root $\alpha_{i}$ is short in $C_{\ell}$. So, the corresponding coroot $\check{\alpha}_{i}$ is long and the inclusion $G_{i}^{\prime} \subset G$ has Rost multiplier 2; see, e.g., [Ga 01b, 2.2] or [M 03, 7.9.2]. Since the Rost invariant has values in $H^{3}\left(k, \boldsymbol{\mu}_{2}^{\otimes 2}\right)$, which is 2-torsion, we get $r_{G}(a)=0$ if $\ell$ is even, and $r_{G}(a)=r_{G_{\ell}^{\prime}}\left(a_{\ell}\right)$ if $\ell$ is odd. In the latter case, applying the formula for groups of type $A$ shows that $t_{R, G}=[Q]$, which is the Tits class of $G$.

\section{$\S 11$. TYPE $E_{6}$}

In this section, we prove the theorem for a group $G$ of type $E_{6}$.

11.1. Groups of inner type $E_{6}$. First, assume $G$ is of inner type; that is, $\Gamma_{k}$ acts trivially on the Dynkin diagram. The center $Z$ is identified with $\boldsymbol{\mu}_{3}$, and we want to prove that $t_{R, G}$ and $t_{G}$ generate the same subgroup of $H^{2}(k, Z)$. Again by Subsection 4.3 , it suffices to consider groups whose Tits index satisfies condition (4.2). Let $G$ be such a group; i.e., we assume that the Tits index of $G$ is

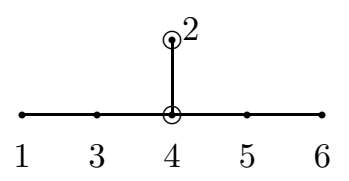

or has more circled vertices. (As in the type $E_{7}$ case in $\S 9$, if more vertices are circled, then $G$ is split.)

We write $G_{1}^{\prime}$ and $G_{5}^{\prime}$ for the components of $G^{\prime}$ corresponding to the subdiagrams with vertices 1,3 and 5,6 , respectively. They are of type $A_{2}$. Moreover, since the fundamental weights $\omega_{1}$ and $\omega_{5}$ have the same class in $\Lambda / \Lambda_{r}$, we may identify $G_{1}^{\prime}$ and $G_{5}^{\prime}$ with $S L(D)$ for some central simple algebra $D$ of degree 3 over $k$. The center of $S L(D)$ is identified with $\boldsymbol{\mu}_{3}$ in a natural way, and the composition

$$
H^{1}\left(k, \boldsymbol{\mu}_{3}\right) \rightarrow H^{1}(k, S L(D))=H^{1}\left(k, G_{i}^{\prime}\right) \stackrel{r_{G_{i}^{\prime}}}{\longrightarrow} H^{3}(k, \mathbb{Q} / \mathbb{Z}(2))
$$

is the cup product with $m[D]$ for $m$ equal to 1 or 2 by Example 2.4.

Comparing the description of the center of $G$ and the $G_{i}^{\prime}$ given by (8.3) and (8.9), we see that the map $Z \rightarrow G_{1}^{\prime} \times G_{5}^{\prime}$ is $\zeta \mapsto\left(\zeta^{2}, \zeta^{2}\right)$. Hence, the induced map $H^{1}(k, Z) \rightarrow$ $H^{1}\left(k, Z_{1}^{\prime}\right) \times H^{2}\left(k, Z_{5}^{\prime}\right)$ is given by $a \mapsto(2 a, 2 a)$. Applying equation (5.8), we see that the composition (1.1) is

$$
a \mapsto 4 a \cdot m[D] \in H^{3}\left(k, \boldsymbol{\mu}_{3}^{\otimes 2}\right) .
$$

But $4 a$ equals $a$ because the exponent of $Z$ is 3 . Therefore, $t_{R, G}$ equals $m[D]$, i.e., generates the same subgroup of $H^{2}(k, Z)$ as $[D]$, which is $t_{G}$ (see [Ti71, p. 211]).

11.2. Groups of outer $E_{6}$ type. Suppose now that the group $G$ is of outer type; i.e., the absolute Galois group acts nontrivially on the Dynkin diagram of $G$. The kernel of the homomorphism $\Gamma_{k} \rightarrow \operatorname{Aut}(\Delta)=\mathbb{Z} / 2$ determines a quadratic extension $L$ of $k$, over which the group becomes of inner type. The center $Z$ of $G$ is isomorphic to $\boldsymbol{\mu}_{3[L]}$. 
By the inner type case, we know that $t_{R, G}-m t_{G}$ is killed by $L$. Since it lives in the group $H^{2}(k, Z)$, which is of exponent 3 , while $L$ is quadratic over $k$, a restriction/corestriction argument shows that $t_{R, G}-m t_{G}$ is zero in $H^{2}(k, Z)$ (see [Brown, 9.5 (ii)]). This concludes the proof for groups of type $E_{6}$.

\section{$\S 12$. TYPE $D_{\ell}$ FOR $\ell$ EVEN} $D_{4}$.

Here we prove the main theorem for groups of type $D_{\ell}$ for $\ell$ even, including trialitarian

12.1. Groups of inner type $D_{\ell}$. As before, first we assume that $G$ is of inner type, so that $Z$ is identified with $\boldsymbol{\mu}_{2} \times \boldsymbol{\mu}_{2}$. We want to prove that the composition (1.1) is the cup product with the Tits class, where the cup product is induced by the bilinear map given in (2.2). By Subsection 4.3, it suffices to consider groups with Tits index satisfying condition (4.2), i.e., with Dynkin diagram

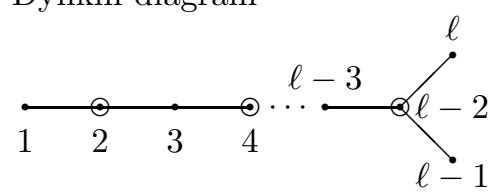

or having more circled vertices.

We write $G_{i}^{\prime}(i=1,3, \ldots, \ell-3, \ell-1, \ell)$ for the component of $G^{\prime}$ corresponding to the vertex $\alpha_{i}$. They are of type $A_{1}$ and may be identified with $S L\left(Q_{i}\right)$ for some quaternion algebras $Q_{i}$. Each has center $Z_{i}^{\prime}$ isomorphic to $\boldsymbol{\mu}_{2}$. Moreover, since $\omega_{1}, \ldots, \omega_{\ell-3}$ and $\omega_{\ell-1}+\omega_{\ell}$ have the same class in $\Lambda / \Lambda_{r}$, we have $Q_{1}=\cdots=Q_{\ell-3}=Q$ for some quaternion algebra $Q$ satisfying $[Q]=\left[Q_{\ell-1}\right]+\left[Q_{\ell}\right]$.

Since $G$ is of inner type, the homomorphisms $z_{0}, z_{1}$ described in Example 8.6 are $k$-defined even though $G$ need not be split. Hence, we get a map

$$
\left(z_{0}, z_{1}\right): \mu_{2} \times \mu_{2} \rightarrow Z \subset Z_{1}^{\prime} \times \cdots \times Z_{\ell-3}^{\prime} \times Z_{\ell-1}^{\prime} \times Z_{\ell}^{\prime}
$$

The induced map $H^{1}(k, Z) \rightarrow \prod_{i} H^{1}\left(k, Z_{i}^{\prime}\right)$ is given by

$$
\left(a_{0}, a_{1}\right) \mapsto\left(a_{0}+a_{1}, \ldots, a_{0}+a_{1}, a_{1}, a_{0}\right) .
$$

Applying equation (5.8) and the formula for the groups of type $A$ (see Example 2.4), we get $r_{G}\left(a_{0}, a_{1}\right)=\frac{\ell-2}{2}\left(a_{0}+a_{1}\right) \cdot[Q]+a_{1} \cdot\left[Q_{\ell-1}\right]+a_{0} \cdot\left[Q_{\ell}\right]$; that is,

$$
r_{G}\left(a_{0}, a_{1}\right)=\left\{\begin{array}{lll}
a_{0} \cdot\left[Q_{\ell-1}\right]+a_{1} \cdot\left[Q_{\ell}\right] & \text { if } \ell \equiv 0 & (\bmod 4), \\
a_{0} \cdot\left[Q_{\ell}\right]+a_{1} \cdot\left[Q_{\ell-1}\right] & \text { if } \ell \equiv 2 & (\bmod 4) .
\end{array}\right.
$$

On the other hand, we have

$$
\omega_{\ell-i}\left(a_{0}, a_{1}\right)=a_{i} \quad \text { and } \quad \omega_{\ell-i}\left(t_{G}\right)=\left[Q_{\ell-i}\right]
$$

for $i=0,1$. Combining (12.2) and (12.3) with the definition of the cup product in (2.2), we conclude that the composition (1.1) is equal to the cup product with the Tits class $t_{G}$.

12.4. Groups of outer $D_{\ell}$ type. The case of groups of type ${ }^{2} D_{\ell}$ can be reduced to the ${ }^{1} D_{\ell}$ case as in [MPT, p. 817]. Alternatively, one can apply the same method as for groups of inner type. In this case, $G^{\prime}$ is a direct product of $(\ell-2) / 2$ copies of $S L(Q)$ and a transfer $R_{L / k} S L\left(Q^{\prime}\right)$, where $Q^{\prime}$ is a quaternion algebra over a quadratic extension $L$ of $k$. To apply (5.8), one needs to know the Rost invariant of $R_{L / k} S L\left(Q^{\prime}\right)$, which was specified in [M 03, 9.8].

It only remains to treat the trialitarian groups, i.e., the groups $G$ of types ${ }^{3} D_{4}$ or ${ }^{6} D_{4}$. Recall what this means. The automorphism group of the Dynkin diagram $\Delta$ of $G$ is the symmetric group on three letters, and the superscript 3 or 6 denotes the size of the image 
of the homomorphism $\Gamma_{k} \rightarrow \operatorname{Aut}(\Delta)$. The kernel of this homomorphism fixes at least one separable cubic field extension of $k$; we pick one and call it $L$. The group $G$ is of type ${ }^{1} D_{4}$ or ${ }^{2} D_{4}$ over $L$. Since the exponent of $Z$ (i.e., 2 ) is relatively prime to the dimension of $L / k$ (i.e., 3 ), the proof can be completed by using a restriction/corestriction argument as for groups of outer type $E_{6}$.

\section{§13. Applichtion to groups of type $E_{7}$}

Now, we apply the main theorem to prove a result for groups of type $E_{7}$. Every simply connected group $G$ of type $E_{7}$ can be described in terms of "gifts" as in [Ga 01a]. A gift is a triple $(A, \sigma, \pi)$, where $A$ is a central simple $k$-algebra of degree $56, \sigma$ is a symplectic involution on $A$, and $\pi: A \rightarrow A$ is $k$-linear and satisfies certain axioms; see [Ga01a, Definition 3.2] for a precise statement. The Brauer class of $A$ is the Tits class $t_{G}$. The $k$-points of $G$ are the elements $a \in A^{\times}$such that $\sigma(a) a=1$ and $\operatorname{Int}(a) \pi=\pi \operatorname{Int}(a)$, where $\operatorname{Int}(a)$ denotes the automorphism $x \mapsto a x a^{-1}$ of $A$.

The associated adjoint group $\bar{G}$ is a subgroup of $P G L(A)$; it has $k$-points $\operatorname{Int}(a)$ for $a \in A^{\times}$such that $\sigma(a) a$ is in $k^{\times}$and $\operatorname{Int}(a) \pi=\pi \operatorname{Int}(a)$. The exact sequence

$$
1 \longrightarrow \mu_{2} \longrightarrow G \longrightarrow \bar{G} \longrightarrow 1
$$

gives a connecting homomorphism

$$
\delta: \bar{G}(k) \rightarrow H^{1}\left(k, \boldsymbol{\mu}_{2}\right)=k^{\times} / k^{\times 2} .
$$

As in [KMRT, §31],

$$
\delta(\operatorname{Int}(a))=\sigma(a) a \in k^{\times} / k^{\times 2} .
$$

If $\lambda k^{\times 2}$ is in the image of $\delta$, then its image in $H^{1}(k, G)$ is zero and the composition (1.1) sends $\lambda$ to zero. The main theorem gives the following.

Corollary 13.1. If $\lambda k^{\times 2}$ is in the image of $\delta$, then $(\lambda) \cdot[A]=0$.

In [Ga 01a, 6.2], this result was observed in the case where $k=\mathbb{R}$.

Acknowledgements. The first author thanks Université Paris 13 for its hospitality while some of the work on this paper was performed.

\section{REFERENCES}

[Borel] A. Borel, Linear algebraic groups, second ed., Graduate Texts in Mathematics, vol. 126, Springer-Verlag, New York, 1991. MR1102012 (92d:20001)

[Bou] N. Bourbaki, Lie groups and Lie algebras: Chapters 4-6, Springer-Verlag, Berlin, 2002. MR1890629 (2003a:17001)

[BS] A. Borel and T. A. Springer, Rationality properties of linear algebraic groups. II, Tohoku Math. J. (2) 20 (1968), 443-497 (= Borel, Oe., vol. 2, \#76). MR0244259 (39:5576)

[BT] A. Borel and J. Tits, Groupes réductifs, Inst. Hautes Études Sci. Publ. Math. 27 (1965), 55-150. MR0207712 (34:7527)

[Brown] Kenneth S. Brown, Cohomology of Groups, Graduate Texts in Mathematics, vol. 87, SpringerVerlag, New York-Berlin, 1982. MR0672956 (83k:20002)

[Ga01a] R. S. Garibaldi, Groups of type $E_{7}$ over arbitrary fields, Comm. Algebra 29 (2001), no. 6, 2689-2710. MR1845137 (2002f:17003)

[Ga01b] The Rost invariant has trivial kernel for quasi-split groups of low rank, Comment. Math. Helv. 76 (2001), no. 4, 684-711. MR1881703 (2003g:20079)

[Gi] Ph. Gille, Invariants cohomologiques de Rost en caractéristique positive, K-Theory 21 (2000), 57-100. MR1802626 (2001k:11064)

[KMRT] M.-A. Knus, A. S. Merkurjev, M. Rost, and J.-P. Tignol, The book of involutions, Colloquium Publications, vol. 44, Amer. Math. Soc., 1998. MR1632779 (2000a:16031)

[M96] A. S. Merkurjev, The norm principle for algebraic groups, St. Petersburg Math. J. 7 (1996), no. 2, 243-264. MR1347513 (96k:20088) 
[M 03] - Rost invariants of simply connected algebraic groups, with a section by S. Garibaldi, in Cohomological invariants in Galois cohomology, University Lecture Series, vol. 28, Amer. Math. Soc., 2003. MR1999385

[MPT] A. S. Merkurjev, R. Parimala, and J.-P. Tignol, Invariants of quasitrivial tori and the Rost invariant, St. Petersburg Math. J. 14 (2003), 791-821. MR1970336 (2004c:11045)

[MT] A. S. Merkurjev and J.-P. Tignol, The multipliers of similitudes and the Brauer group of homogeneous varieties, J. Reine Angew. Math. 461 (1995), 13-47. MR1324207 (96c:20083)

[SS] T. A. Springer and R. Steinberg, Conjugacy classes, Seminar on Algebraic Groups and Related Finite Groups (The Institute for Advanced Study, Princeton, N.J., 1968/69), Springer, Berlin, 1970, pp. 167-266. MR0268192 (42:3091)

[St] R. Steinberg, Lectures on Chevalley groups, Yale University, New Haven, Conn., 1968. MR0466335 (57:6215)

[Ti66] J. Tits, Classification of algebraic semisimple groups, Algebraic Groups and Discontinuous Subgroups, Proc. Symp. Pure Math., vol. IX, AMS, 1966, pp. 32-62. MR0224710 (37:309)

[Ti 71$] ـ$ Représentations linéaires irréductibles d'un groupe réductif sur un corps quelconque, J. Reine Angew. Math. 247 (1971), 196-220. MR0277536 (43:3269)

Department of Mathematics \& Computer Science, Emory University, Atlanta, Georgia 30322

E-mail address: skip@member.ams.org

$U R L:$ http://www.mathcs.emory.edu/〜 skip/

Laboratoire Analyse, Géométrie \& Applications, UmR CnRS 7539, Institut Galilée, UniVersité Paris 13, 93430 Villetaneuse, France

E-mail address: queguin@math.univ-paris13.fr

$U R L:$ http://www-math. univ-paris13.fr/ queguin/

Received 27/JUL/2006

Originally published in English 